\title{
Orthotopic testicular transplantation in mice
}

\author{
Jianhui Li ${ }^{1,2}$, Hannu Savolainen ${ }^{3}$, FuQing $\operatorname{Tan}^{4}$ and Shusen Zheng ${ }^{1}$ \\ ${ }^{1}$ Key Laboratory of Organ Transplantation, Key Laboratory of Combined Multi-organ Transplantation, Department of \\ Hepatobiliary and Pancreatic Surgery, Ministry of Public Health, Medical College, First Affiliated Hospital, Zhejiang \\ University, Hangzhou 310003, Zhejiang Province, People's Republic of China, ${ }^{2}$ Service de Médecine Intensive Adulte \\ et Division de Physiopathologie, CHUV-1010 Lausanne, Switzerland, ${ }^{3}$ Department of Cardiovascular Surgery, \\ University Hospital, 3010 Berne, Switzerland and ${ }^{4}$ Department of Urology, College of Medicine, the First Affiliated \\ Hospital, Zhejiang University, Hangzhou, Zhejiang 310003, People's Republic of China
}

Correspondence should be addressed to S Zheng; Email: surgeonlee@hotmail.com

\begin{abstract}
A method of revascularized orthotopic testicular transplantation (OTT) was developed in mice. The left testis was selected as donor graft for the operation due to less variation in anatomy. There were three groups: 1) a control group (n=24), 2) a group of castrated mice $(n=24)$, and 3) a group in which OTT $(n=24)$ was performed. Morphologically, the transplanted testes showed active spermatogenesis and normal structure of epididymis at 4 and 5 weeks. The function of the transplants was examined by RIA at designed time points. $\mathrm{LH}, \mathrm{FSH}$, and testosterone showed return to normal levels at 4 weeks. To our knowledge, this is the first report of successful revascularized OTT in mice. The model may prove useful in research in reproductive medicine, especially using knockout and transgenic mice.

Reproduction (2010) 139 447-452
\end{abstract}

\section{Introduction}

The testis has been of great interest in the research of endocrine regulation of spermatogenesis as well as privileged immune sites (Schuppe \& Meinhardt 2005, Elizabeth 2006). The relationship between the functions still remains unexplained (Nasr et al. 2005).

Transplantation of spermatogonial cells and testicular tissue using transgenic mouse strains provides a possibility to address these mechanisms. The difficulty of restoring fertility in the cells is a significant practical problem (Clouthier et al. 1996, Cooke \& Saunders 2000, Kanatsu-Shinohara et al. 2004, Dobrinski \& Rathi 2008). Nonvascularized techniques are different from the normal physiological situation. Transplantation of a revascularized organ may be a viable option. Testis transplantation model in mice has been considered less mainly due to the high variability of the vessel anatomy and the delicate microsurgical technique required (Kirpatovskii et al. 1977, Setchell 1990, Barten \& Newling 1996). Based on our previous experience in the rat (Tan et al. 2005, 2006, Zhang et al. 2005), we now present a novel technique for mouse-to-mouse orthotopic testicular transplantation (OTT) with associated histological and laboratory chemical findings showing normal function after the operation.

\section{Results}

Anatomical variance of testicular vessels

The anatomic variances of the spermatic vein were similar in both the right and the left testis $(n=22)$. In $81.8 \%$, the testicular artery originated directly from the aorta. In $63.6 \%$, the right testicular artery originated from the aorta by crossing the inferior vena cava (IVC; Fig. 1).

\section{Operating time}

Total operating time was $3 \mathrm{~h}$ (graft harvest $57 \pm 5 \mathrm{~min}$, cold ischemia $17 \pm 2 \mathrm{~min}$, warm ischemia and completion of anastomoses $70 \pm 4 \mathrm{~min}$, value $=$ mean \pm S.E.M., $n=24$ ).

\section{Graft histopathology}

Macroscopically, the transplants showed adequate blood supply with normal weight and size when checked post operation (Figs $2 \mathrm{~F}$ and $3 \mathrm{~A}$ and $\mathrm{B}$ ). Morphologically, the grafts showed active spermatogenesis at 4 and 5 weeks after transplantation. The seminiferous tubules of the transplanted testes were intact and consisted of Sertoli cells and well-arranged spermatogenic cells. The Sertoli cells and spermatogonia 


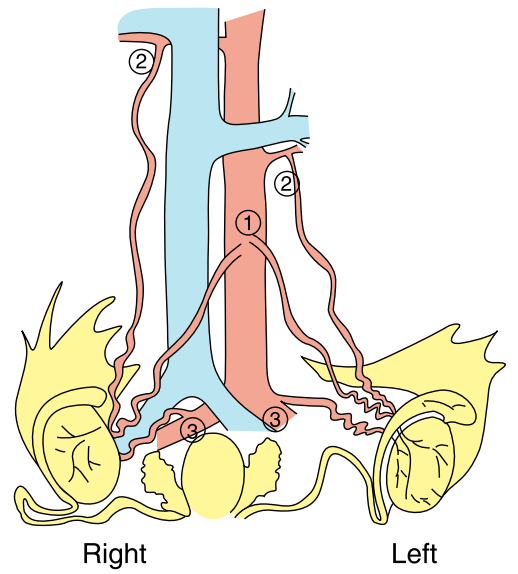

A

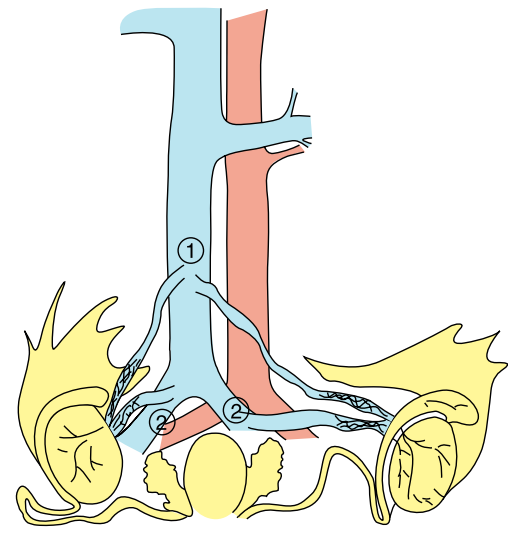

Right
Left

Figure 1 (A) Variability of testicular artery anatomy $(n=22) .1$, Testicular artery originates directly from the aorta (left: $81.8 \%$, right: $63.6 \%$ ); 2, testicular artery originates from the kidney artery (left: $13.6 \%$, right: $13.7 \%) ; 3$, testicular artery originates from the iliac artery (left: $4.6 \%$, right: $22.7 \%$ ). (B) Variability of spermatic vein anatomy $(n=22) .1$, Spermatic vein drains into the inferior vena cava (left: $13.6 \%$, right: $9.1 \%$ ); 2 , spermatic vein drains into the iliac vein (left: $86.4 \%$, right: $90.9 \%$ ); $1+2$, spermatic vein drains into the inferior vena cava and iliac vein (left: $13.6 \%$, right: $4.5 \%$ ).

lined at the basal membrane, while the spermatocytes, round and elongated spermatids were closely associated with the Sertoli cells. There were spermatids (round and elongating) about to be released into the lumen as well as some which already had done so. The lumen was filled with mature spermatozoa. Leydig cells and peritubular cells were arranged around the tubules. The epididymis of the grafts showed round or elongated tubules and intertubular compartments. Each tubule was lined with columnar epithelium. The lumen was packed with spermatozoa. The intertubular components were made of connective tissue, predominantly collagen fibers. No obvious difference was seen in comparison with the control testes (Fig. 3C-J).

\section{Laboratory analyses}

The function of the tissue was investigated by RIA of hormones in all groups. The level of serum testosterone decreased during the first week after the transplantation, but it slowly recovered to the same levels as in the intact group. In the castrated group, the testosterone level remained low. The $\mathrm{LH}$ and FSH levels increased slightly during the first 2 weeks after operation and returned to normal levels by the end of the fourth week. The castrated group showed marked elevation of $\mathrm{LH}$ and FSH levels (Fig. 4).

\section{Discussion}

This study was conducted to develop a model of revascularized OTT, which could provide an opportunity for new applications in reproductive medicine and conservation biology with knockout and transgenic mice.

\section{Technique of orthotopic testicular transplantation in mice}

During pretransplantation anatomical investigation, we observed that spermatic vein anatomy is similar on both sides (Fig. 1B). The arterial supply showed variations with $63.6 \%$ of the right testicular artery
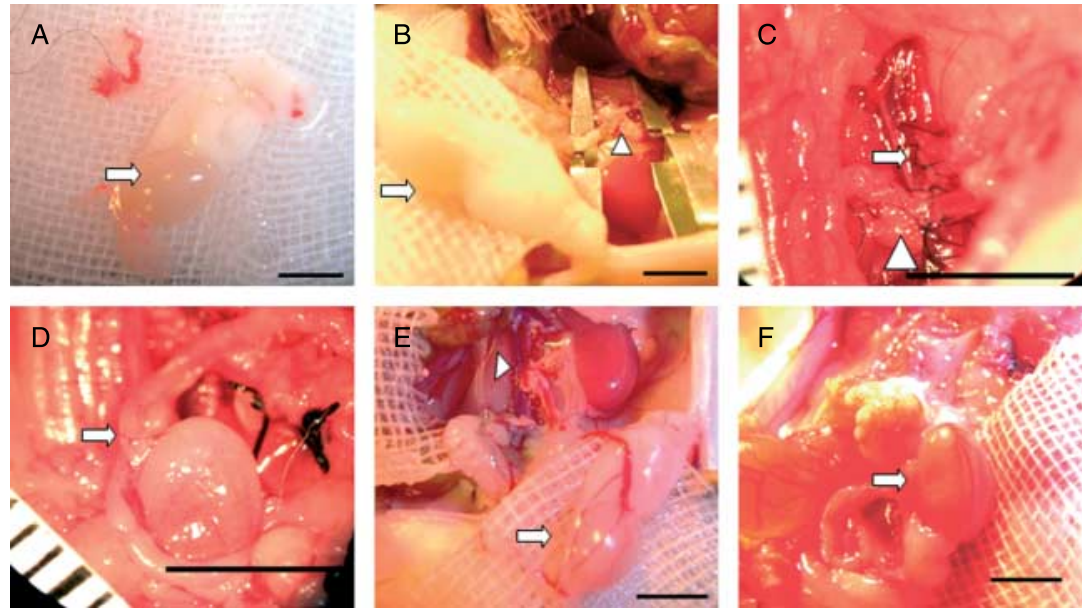

Figure 2 (A) Preparation and perfusion of donor testis (arrow). (B) The graft (arrow) was implanted into the recipient; the arrowhead indicates the anastomosis site of the vessels. (C) The donor aorta was anastomosed end-to-side to the recipient aorta using interrupted suture techniques (arrow) and an end-to-side anastomosis completed with continuous suture techniques for the vein (arrowhead). (D) The donor testicles were removed, and a vasovasostomy was performed with 11-0 nylon using one-layer technique (arrow). (E) Immediately observation of the graft (arrow), following removing the clamp of aorta and vena cava at the anastomosis site (arrowhead). (F) Appearance of the graft 5 weeks after surgery with normal blood supply and size (arrow), bar, $5 \mathrm{~mm}$. 
A
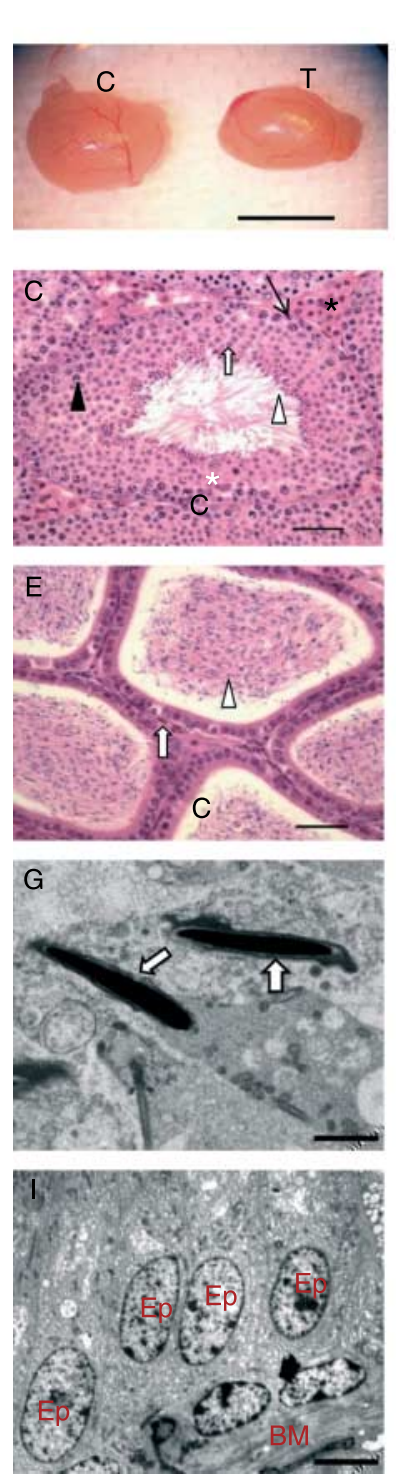

Figure 3 (A) At 5 weeks after surgery, macroscopic observation of the isotransplanted testes ( $\mathrm{T}$, transplantation; $\mathrm{C}$, control; bar, $5 \mathrm{~mm}$ ). (B) Ratio of the weight of testes to body weight at 5 weeks after surgery (data are means \pm s.E.M., $P>0.05$ versus control). (C-F) Light microscopic examination in isotransplanted mice. (C-D) The grafts showed normal spermatogonia (black arrows), spermatocytes (black arrowheads), spermatids (white arrows), spermatozoa (white arrowheads), Sertoli cells (white asterisks), and Leydig cells (black asterisks) in seminiferous tubules. (E-F) The corpus part of the epididymis of the grafts showed round or elongated tubules and intertubular compartments. Each tubule was lined with columnar epithelium cells (white arrows), with spermatozoa (white arrowheads) packed in the lumen (H\&E; T, transplantation; C, control; bar, $50 \mu \mathrm{m}$ ). (G-J) Transmission electron microscopic observation of the graft 4 weeks after surgery. (G) Well-developed elongated spermatids show regular and condensed nuclei in seminiferous tubules (white arrows; bar, $2 \mu \mathrm{m}$ ). (H) Intact blood-testis barrier (black arrows; bar, $0.5 \mu \mathrm{m}$ ). (I) Normal structure of basement membrane (BM) of epididymis and epididymal epithelium (Ep); bar, $5 \mu \mathrm{m}$. (J) Well-developed Sertoli cells (Se) and spermatocytes (Sp); bar, $2 \mu \mathrm{m}$. originating directly from the aorta, then crossing the IVC (Fig. 1A). We felt this would increase the technical difficulty and risk of damage of the testicular artery when dissecting the vessels and decided to use the left testicle instead.

In our initial experience, thrombus formation at anastomotic site was the main reason for failure. In order to avoid it, we used a patch of the aortic wall and a long segment of the vena cava from the donor, and performed an end-to-side anastomosis with the aorta and IVC. Using this technique makes the dissecting as well as completing the anastomosis easier. It also saves operating time (Fig. 5).

The small diameter of the testicular artery limits direct flushing of the organ through the lumen of the artery. Flushing through this route tends to cause intimal lesions leading to thrombosis. Therefore, we used a 27-gauge needle (smooth tip) placed and fixed in the lumen of the aorta instead. The organ was gently flushed with cold heparinized Ringer's lactate until the graft becomes uniformly pale. Care should be taken to avoid formation of air bubbles during flushing.

The mouse testis is extremely sensitive to ischemia (Attaran \& Hodges 1966, Goldstein et al. 1983). Minimizing this is the key to success in testicular transplantation in mice. More than $2 \mathrm{~h}$ of ischemia almost invariably leads to loss of graft. To reduce the ischemia time, we simultaneously prepared the recipient and the graft itself. This effectively decreased ischemia time and kept our total recipient operating times at $<70 \mathrm{~min}$.

Hypothermia is required during transplantation to suppress metabolism and catabolic enzymes (Jamieson \& Friend 2008). We used cold heparinized Ringer's lactate. During implantation, we covered the graft between two layers of gauze and continuously flushed the surface with cold Ringer's lactate in order to protect it until full blood supply was re-established.

\section{Graft injury and function after transplantation}

The criterion for a well-vascularized graft is a coiling vascular pattern beneath the tunica albuginea after release of clamps (Lee et al. 1999, Zhang et al. 2005, Tan et al. 2006).

Postoperatively, we observed that the testosterone levels in the recipients decreased during the first 2 weeks but then recovered to normal levels by 4 weeks. The $\mathrm{LH}$ and FSH levels slightly increased during the first 2 weeks after transplantation but returned to normal levels by the fourth week indicating recovery from intraoperative ischemia reperfusion injury.

Macroscopically, the grafts were well vascularized, with normal size and weight compared to the control. Morphologically, the transplanted grafts showed active spermatogenesis without obvious graft rejection. This is in line with our previous studies in rats (Zhang et al. 2005). In recent years, spermatogonial cells and 

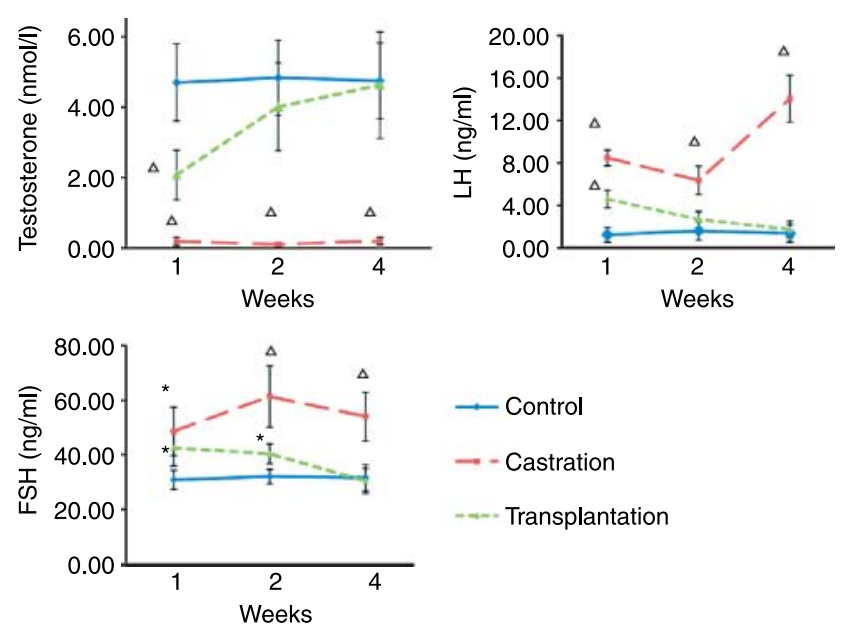

Figure 4 Serum hormone (testosterone, LH, and FSH) levels in intact mice or in bilateral castrated mice and the orthotopically transplanted mice. Each data point in the figure represents the mean \pm s.E.M. of hormone levels of six mice. In transplanted recipients, the level of serum testosterone decreased in the first weeks, but slowly recovered to the normal level compared with the intact group, while in the bilateral castrated group the testosterone remained at the low level from the first week. Both $\mathrm{LH}$ and FSH levels in the transplanted group slightly increased in the first and second weeks after operation compared with the intact group, but in the third week returned to the normal level respectively, while the bilateral castrated groups showed marked elevation of levels of LH and FSH (white arrowheads indicate $P<0.01$ compared with control group, and the asterisks indicate $P<0.05$ compared with control group).

testicular tissue from the mouse, hamster, pigs, goats, monkeys, and human have been transplanted to an ectopic location of mice (i.e. under the skin on the back; Honaramooz et al. 2002, Schlatt et al. 2002, Dobrinski 2005) with the spermatogenesis having been maintained. The technique has been introduced as a strategy to investigate testicular development and preserve fertility in mammals (Schlatt et al. 2002). Heterotopic transplantation of testes provides the organ structure and cell components for normal spermatogenesis, but the grafted tissues lack the original vascular environment. Compared to heterotopic testicular germ cells and tissue transplantation, the OTT may have the advantage that both the reproductive tract and the blood vessels are similar to the anatomy in situ and the physiological microenvironment. Spermatogenesis is a complex process in vivo, regulated by endocrine and intratesticular factors, and it is affected by temperature (Wakayama \& Iseki 2009) as well. The vascularized transplantation of an intact testis provides sufficient testosterone and the physiological microenvironment to maintain spermatogenesis. Clinically, vascularized testicular transplantation has the potential ability of becoming an effective way to treat male gonadal dysfunction (i.e. intra-abdominal cryptorchidism) and male infertility (Zhang et al. 2005).
To our knowledge, this is the first report of successful OTT in mice with proven function. The model may be useful in research of transplantation immunology, fertility, and spermatogenesis.

\section{Materials and Methods}

\section{Animals}

BALB/c mice (25-28 g) were used. Both donor and recipient surgeries were performed on mice anesthetized with pentobarbital (priming bolus $75 \mathrm{mg} / \mathrm{kg}$ i.p., diluted w/saline to $6 \mathrm{mg} / \mathrm{ml}$ plus infusion s.c. at about $20 \mathrm{mg} / \mathrm{kg}$ per hour to maintain anesthesia), which provides good recovery after surgery. A heating pad was used during the operation to maintain body temperature. The study was performed in accordance with National Institutes of Health guidelines for the use of experimental animals (NIH Publication no. 85-23, revised 1996) and with the approval of the animal research committee at our institutions.

\section{Experimental groups}

Initial experiments were performed to investigate the anatomical variability of testicular vessels in the mouse strain used $(n=22)$. The mice were also used for operation practice to refine the technique. In the actual experiment, the mice were divided into three groups: 1$)$ a control group $(n=24), 2)$ a group of castrated mice $(n=24)$, and 3$)$ a group in which OTT $(n=24)$ was performed.

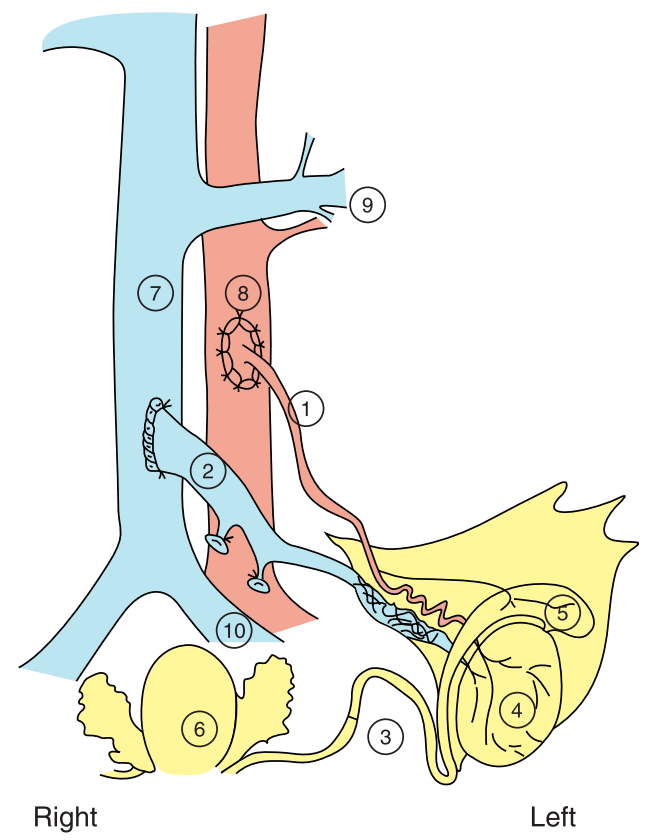

Figure 5 Schematic drawing of anatomy of the abdomen and orthotopic testicular transplantation in mice. 1 , Testicular artery; 2 , spermatic vein with part of the vena cava of donor; 3 , vas deferens; 4 , testis; 5 , caput epididymids of donor testis; 6 , bladder; 7 , vena cava; 8 , aorta; 9 , vessels of left kidney; 10, common iliac vessels. 


\section{Donor preparation}

The mice were anesthetized and placed in supine position with their limbs immobilized. The skin was prepared and sterilized. A long midline incision was made. Upon entering the abdomen, an operating microscope with $\times 16-20$ magnification (OME-JA, Olympus Inc., Tokyo, Japan) was used, the abdominal wall was retracted, and the bowel was reflected to the left. As the anatomy of testicular vessels is highly variable and their caliber minute, a long segment of aorta and IVC was grafted along with the testicular vessels. Branches of the aorta and IVC from the level of renal vein were isolated from connective tissues and ligated. The left iliac vessels distal to the ostium of the testicular vein were doubly ligated and cut. After gently isolating the aorta and the vena cava, a 5-mm segment of aorta was freed above and below the origin of the donor testicular artery. After that, the vena cava was transected below the iliolumbar vein. The aortic segment was ligated. A syringe (27-G needle, smooth tip) was inserted through an incision in the anterior wall of the aorta and secured with 6-0 silk. The graft was gently flushed via the testicular artery with heparinized Ringer's lactate $(50 \mathrm{IU} / \mathrm{ml}$ heparin) until the graft became pale and the fluid from the testicular vein became clear. The vas deferens was transected at the middle third. Then, a 2-mm patch of gubernaculum ligament-scrotal epithelium was cut and the graft immersed at $4{ }^{\circ} \mathrm{C}$ in heparinized Ringer's lactate (Figs 2A and 5).

\section{Graft preparation}

The donor testis was placed on gauze and kept in cold $\left(4{ }^{\circ} \mathrm{C}\right)$ in Ringer's lactate. During this preparation, the aorta and vena cava were cleaned from extra vascular tissue and the cut ends irrigated with heparinized Ringer's lactate. The aortic segment including the testicular artery was longitudinally incised toward the ostium of the artery with fine scissors. The incised wall of the aorta was trimmed to an oval-shaped patch with the center of the ostium of the testicular artery (Figs 2A and 5).

\section{Recipient preparation and transplantation}

Surgery was performed through a midline laparotomy. The abdominal wall was retracted and the bowel was reflected to the right. Upon entering the abdomen, the aorta and IVC were freed and mobilized below the left renal vein and occluded with two microvascular clamps (8 mm long; Roboz Surgical Instruments, Rockville, MD, USA), one at each end. Two longitudinal incisions $(0.5-0.6 \mathrm{~mm})$ were made with fine needle and scissors, and the proximal cut ends were irrigated with heparinized Ringer's lactate. The graft was implanted into the recipient. The donor aorta was anastomosed end-to-side to the recipient aorta using interrupted suture techniques with 11-0 nylon. A venotomy was performed in the anterior wall above the microvessel clip, and an end-to-side anastomosis was completed with continuous suture techniques with 11-0 nylon. The graft was then reperfused by removing the two microvascular clamps. The two testes of the recipient were removed, and a vasovasostomy was performed with 11-0 nylon using one-layer technique. The scrotal epithelia were sutured, allowing the graft to be pulled toward the scrotal sac (Figs 2B-E and 5).

To castrate the mice in the comparison group, the testicular vessels and vas deferens were ligated near the testicular hilum, and both testes were then removed.

Postoperatively, the animals were kept warm and allowed to eat and drink at will. Normal saline, 1-2 ml, was injected s.c. if blood loss was estimated to be more than $0.5 \mathrm{ml}$. Graft function was initially monitored by daily palpation.

\section{Morphological investigation}

The mice were anesthetized and a midline laparotomy was performed. The animals were killed on day 7, 14, 28, and 35. The transplanted testes and reproductive tracts were removed and fixed in Bouin's fluid overnight and embedded in paraffin. The tissues were prepared as 4-mm serial sections and stained with hematoxylin and eosin. The stained slides were examined under a light microscopy (Olympus B 50). Another graft segment was cut into $1 \mathrm{~mm}^{3}$ of size, then fixed with $2.5 \%$ glutaraldehyde in $0.1 \mathrm{M}$ phosphate buffer $(\mathrm{pH} \mathrm{6.9)}$ for $2 \mathrm{~h}$ at $4{ }^{\circ} \mathrm{C}$ before post fixation in $2 \%$ osmium tetroxide in the same buffer for $1 \mathrm{~h}$. Samples were dehydrated through ascending grades of ethanol and embedded in Epon. Sections were examined under a transmission electron microscope (Leica-Stereoscan 260, Cambridge, UK).

\section{Determinations of testosterone, $\mathrm{LH}$, and $\mathrm{FSH}$}

At the time of killing, blood was collected and serum was separated, then frozen $\left(-20^{\circ} \mathrm{C}\right)$ until assayed for the levels of LH, FSH, and testosterone (Depu Inc., Tianjin, People's Republic of China). The sensitivities for $\mathrm{LH}, \mathrm{FSH}$, and testosterone assays were $0.15 \mathrm{ng} / \mathrm{ml}, 1.16 \mathrm{ng} / \mathrm{ml}$, and $0.3 \mathrm{nmol} / 1$ respectively. The inter- and intra-assays coefficients of variation were $6-9$ and $8-10 \%$ respectively. All samples were run in duplicate and assayed.

\section{Statistical analysis}

Results are reported as mean \pm S.E.M. Statistical significance among groups was determined by ANOVA followed by Dunnett's test. Probability values of $P<0.05$ were considered significant.

\section{Declaration of interest}

There is no conflict of interest that could be perceived as prejudicing the impartiality of the research reported.

\section{Funding}

This work was supported by National Program on Key Basic Research Project (973. Program) of China; grant number: 2003 CB515501. 


\section{Acknowledgements}

The authors thank Dr Sun Ke (Department of Pathology, First Affiliated Hospital of Zhejiang University), Dr Yilei Zhao (Department of Radiology, First Affiliated Hospital of Zhejiang University), Dr Haiyang Xie, and Dr Lin Zhou (Department of Hepatobiliary and Pancreatic Surgery, First Affiliated Hospital of Zhejiang University) and Mrs Wang Li (Electron Microscopy Unit, Department of Anatomy and Cell Biology, Zhejiang University) for their technical assistance during this study.

\section{References}

Attaran S \& Hodges CV 1966 Technique for testicular transplants. Investigative Urology 3 390-394.

Barten EJ \& Newling DW 1996 Transplantation of the testis; from the past to the present. International Journal of Andrology 19 205-211.

Clouthier DE, Avarbock MR, Maika SD, Hammer RE \& Brinster RL 1996 Rat spermatogenesis in mouse testis. Nature 381 418-421.

Cooke HJ \& Saunders PT 2000 Germ cell transplantation - a fertile field. Nature Medicine 6 16-17.

Dobrinski I 2005 Germ cell transplantation. Seminars in Reproductive Medicine 23 257-265.

Dobrinski I \& Rathi R 2008 Ectopic grafting of mammalian testis tissue into mouse hosts. Methods in Molecular Biology 450 139-148.

Elizabeth S 2006 A historical perspective on immunological privilege. Immunological Reviews 213 12-22.

Goldstein M, Phillips DM, Sundaram K, Young GP, Gunsalus GL, Thau R \& Bardin CW 1983 Microsurgical transplantation of testes in isogenic rats: method and function. Biology of Reproduction 28 971-982.

Honaramooz A, Snedaker A, Boiani M, Scholer H, Dobrinski I \& Schlatt S 2002 Sperm from neonatal mammalian testes grafted in mice. Nature 418 778-781.

Jamieson RW \& Friend PJ 2008 Organ reperfusion and preservation. Frontiers in Bioscience 13 221-235.
Kanatsu-Shinohara M, Toyokuni S \& Shinohara T 2004 Transgenic mice produced by retroviral transduction of male germ line stem cells in vivo. Biology of Reproduction 71 1202-1207.

Kirpatovskii ID, Vasil'ev VI, Smirnova ED, Revazov VG \& Chinilin NF 1977 Microsurgical technics in transplantation of the testis. Khirurgiia 8 72-78.

Lee S, Wang Y, Kim S, Cho C, Tarin T, Sileshi B, Yoon JH, Mazzoni G, Youngkin T, Wolf $\mathbf{P}$ et al. 1999 Mouse-to-rat testicle transplantation. Microsurgery 19 66-70.

Nasr IW, Wang Y, Gao G, Deng S, Diggs L, Rothstein DM, Tellides G, Lakkis FG \& Dai Z 2005 Testicular immune privilege promotes transplantation tolerance by altering the balance between memory and regulatory T cells. Journal of Immunology 174 6161-6168.

Schlatt S, Kim SS \& Gosden R 2002 Spermatogenesis and steroidogenesis in mouse, hamster and monkey testicular tissue after cryopreservation and heterotopic grafting to castrated hosts. Reproduction 124 339-346.

Schuppe HC \& Meinhardt A 2005 Immune privilege and inflammation of the testis. Chemical Immunology and Allergy $\mathbf{8 8}$ 1-14.

Setchell BP 1990 The testis and tissue transplantation: historical aspects. Journal of Reproductive Immunology 18 1-8.

Tan FQ, Chen ZD \& Li JH 2005 Three-cuff method for establishing an allogeneic testis transplantation model in the rat. Zhonghua Nan Ke Xue 11 594-597.

Tan FQ, Chen ZD, Zheng XY, Sun K \& Li JH 2006 Establishment of testis transplantation model and study on mechanism of graft injury in rats. Zhejiang Da Xue Xue Bao Yi Xue Ban 35 297-302.

Wakayama T \& Iseki S 2009 Role of the spermatogenic-Sertoli cell interaction through cell adhesion molecule-1 (CADM1) in spermatogenesis. Anatomical Science International 84 112-121.

Zhang WX, Tan FQ, Wang JX, Chen ZD \& Zheng S 2005 A novel method for orthotopic testicular transplantation in rats. Chinese Medical Journal 118 850-853.

Received 7 November 2008

First decision 27 January 2009

Revised manuscript received 6 November 2009

Accepted 13 November 2009 